\title{
Malignant Peritoneal Mesothelioma Presenting as Ascites of Unknown Origin
}

\author{
Sze Li Siow*, Hans Alexander Mahendran \\ Department of Surgery, Sarawak General Hospital, Malaysia \\ *Corresponding author: szeli18@yahoo.com
}

Received January 21, 2015; Revised February 22, 2015; Accepted March 01, 2015

\begin{abstract}
Malignant mesothelioma is a rare but highly aggressive and fatal tumour arising from the mesothelial cells, which is associated with an involvement of the peritoneum in $30 \%$ of cases. We report a 48-year-old man with malignant peritoneal mesothelioma who presented with ascites of unknown origin, and discussed the clinical presentation, investigation and management, as well as the diagnostic difficulties in managing this unusual and unfortunate case.
\end{abstract}

\section{Keywords: Mesothelioma, peritoneal mesothelioma, ascites}

Cite This Article: Sze Li Siow, and Hans Alexander Mahendran, "Malignant Peritoneal Mesothelioma Presenting as Ascites of Unknown Origin.” American Journal of Medical Case Reports, vol. 3, no. 4 (2015): 98101. doi: 10.12691/ajmcr-3-4-3.

\section{Introduction}

Malignant mesothelioma is a highly invasive and invariably fatal tumor arising from the mesothelial cells that form the serosal lining of the pleural space, pericardium, peritoneal cavity, and the tunica vaginalis [1]. Approximately $70 \%$ of malignant mesotheliomas are pleural in origin; with the remaining $30 \%$ are of peritoneal origin [2]. Both are related to asbestos exposure, with peritoneal mesotheliomas having a shorter latent period of about 20-30 years compared to 30-40 years in pleural mesotheliomas [3]. Here, we discuss a case of malignant peritoneal mesothelioma (MPM) and provide brief review of literature.

\section{Case Report}

A 48-year-old gentleman was admitted to our hospital with complaints of abdominal distension, anorexia and weight loss for one month and was cachexic on admission. He denied any episodes of nausea, vomiting or fever. Physical examination showed a distended abdomen with gross ascites and a right groin swelling. The rest of the examinations including rectal examination were normal and he was not jaundiced. His medical history included diabetes mellitus, dyslipidaemia and hypothyroidism, all being treated accordingly. Family history involved a sister currently dealing with breast cancer. Socially, he was an odd job worker, working daytime in the farm and nighttime as security guard. He lived in a wooden house with asbestos roofing for more than a year about 20 years ago. Biochemical markers for hepatic, cardiac and renal function were within normal limits, while serology for Hepatits B \& $\mathrm{C}$ was negative. Tumour marker carcinoembryonic antigen
(CEA) and carbohydrate antigen (CA) 19-9 were normal. Ultrasound and computed tomography (CT) scan confirmed gross ascites but the liver appeared unremarkable and no other cause for ascites could be identified (Figure 1). Analyses of aspirated ascites for acid-fast-bacilli and malignant cells were negative. Subsequent oesophagogastroduodenoscopy and colonoscopy were performed only showing antral erosion. Since symptoms persisted without a definitive diagnosis, a diagnostic laparoscopy was eventually undertaken. This revealed a diffuse multiple peritoneal and omental nodules. A total of 18.5 litre of fluid were drained and biopsies from peritoneum and omentum were taken. Histopathological and immunohistochemical investigation confirmed mesothelioma of the epithelioid type (Figure 2 \& Figure 3). A diagnosis of diffuse MPM was made. Cytoreduction surgery was not performed as the disease was diffuse and advance, and hyperthermic intra-peritoneal chemotherapy was not available in our institution. Palliative systemic chemotherapy was administered. However, the patient succumbed to the disease within one month of starting chemotherapy.

\section{Discussion}

MPM is a tumor with substantial phenotypic variability and is classified according to the relative proportion of epithelial cells to spindle cells [4]. There are three major histological types which are the epithelioid, sarcomatoid, and mixed (biphasic) types [4]. Epithelioid mesothelioma is the most common. There are several immunohistochemical markers useful for diagnosing mesothelioma but are only accurate when used together as a panel, rather than individually. According to the consensus from the International Mesothelioma Interest Group, diagnosis of mesothelioma is made in $95 \%$ of the time when a panel of 
histochemical markers with at least $80 \%$ sensitivity are chosen for testing [5]. Malignant MPM is characterized by positive staining for epithelial membrane antigen (EMA), calretinin, Wilm tumour 1 antigen (WT1), cytokeratin (CK) 5/6, antimesothelial cell antibody-1, and mesothelin [2]. As for our patient, EMA and Vimentin which were strongly positive were used to differentiate malignant peritoneal mesothelioma (MPM) from reactive mesothelial hyperplasia. In addition, positive mesothelial markers (Calretinin, CK 5/6, Hector Battiflora Mesothelial cell (HBME)-1) in combination with negative epithelial markers (Carcinoembryonic antigen (CEA), CK 20, Thyroid transcription factor (TTF)-1) were used to distinquish mesotheliomas from the metastatic carcinoma to the peritoneum such as colorectal adenocarcinoma.

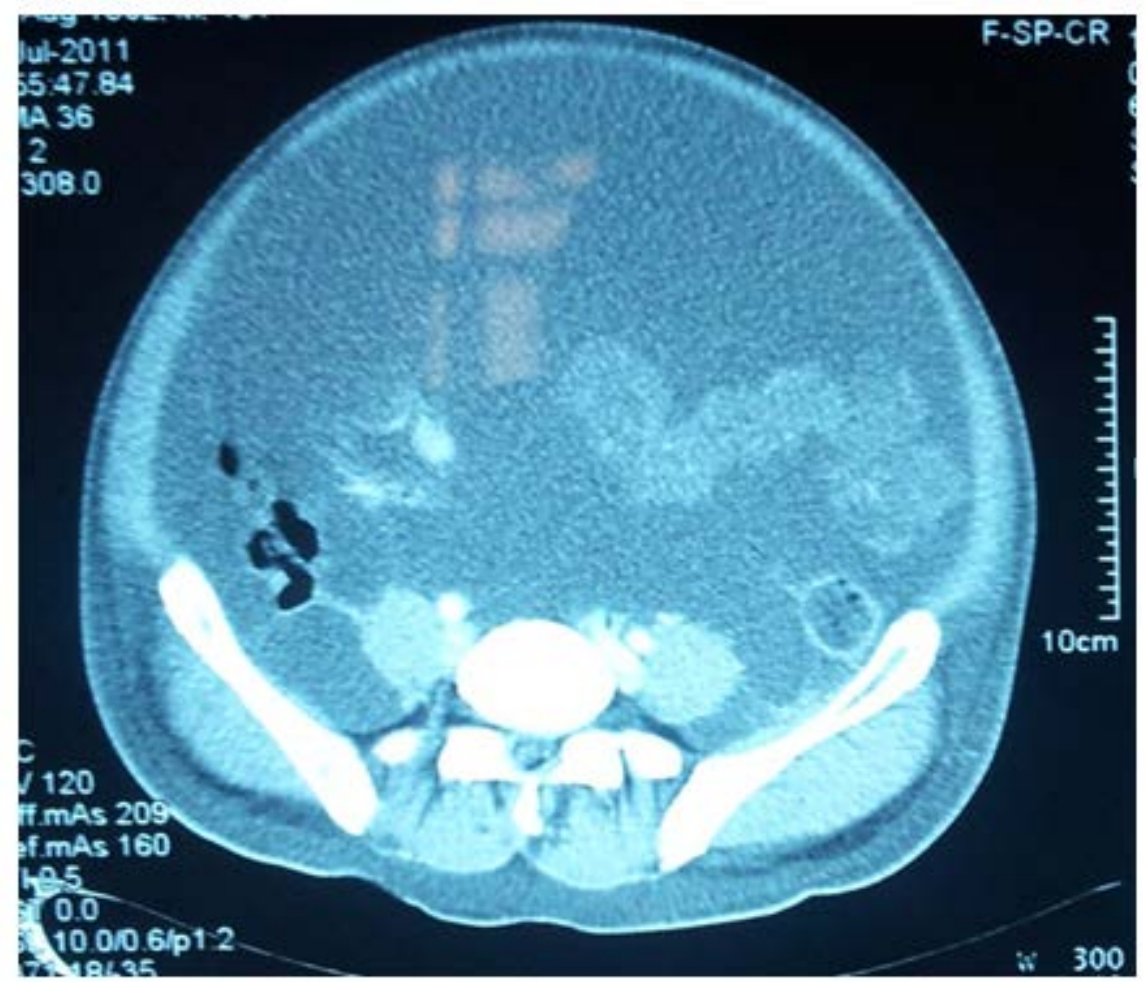

Figure 1. Computed tomography (CT) scans of the abdomen and pelvis showing gross ascites without obvious pathology

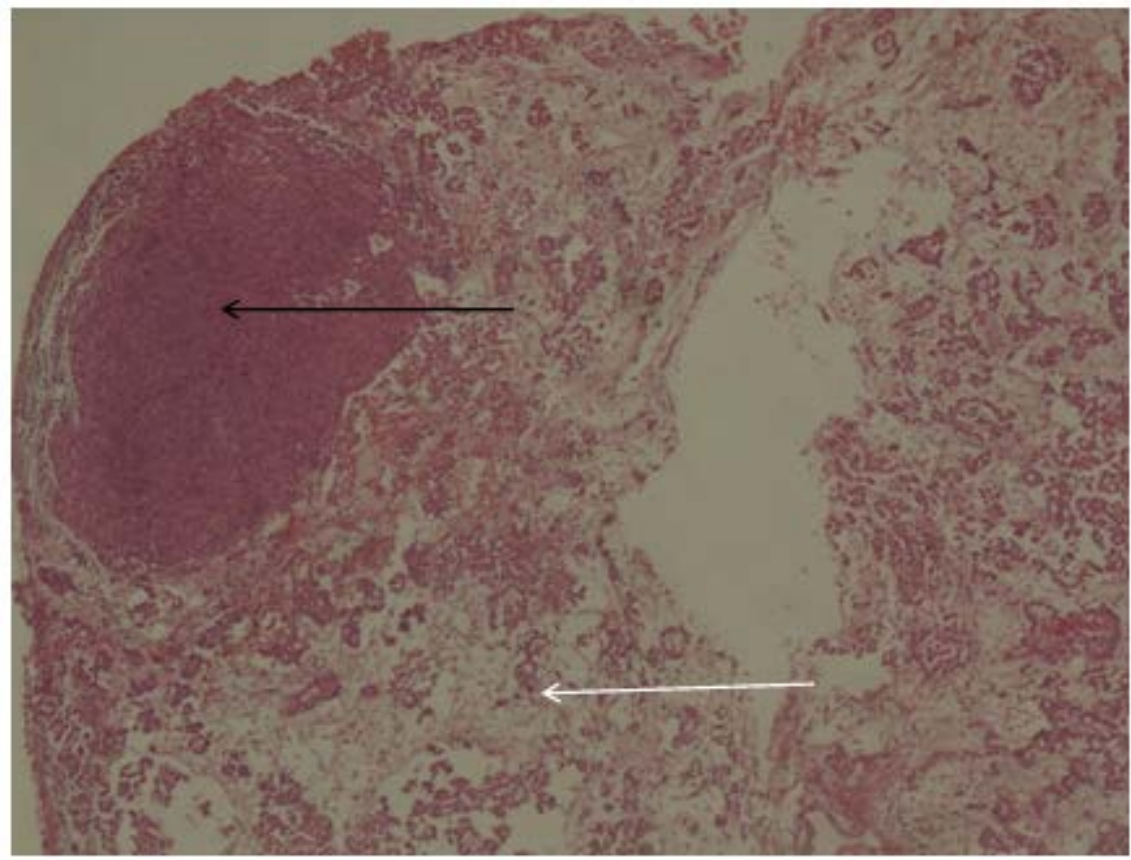

Figure 2. Histopathology: Diffuse MPM-epithelioid type characterized by malignant mesothelial cells in papillary (white arrow) and nodular (black arrow) patterns, exhibiting epithelioid in shape having large round vesicular nuclei, prominent eosinophilic nucleoli and fairly abundance acidophylic cytoplasm

The presentation is often late as early nonspecific symptoms are often dismissed. A study performed at the Washington Cancer Institute, a leading center in treatment of peritoneal mesothelioma, showed that patients typically presented with nonspecific symptoms with the most frequently reported initial symptoms being either 
abdominal pain (33\%) or increasing abdominal girth (31\%) [6]. Routine laboratory tests are often not useful in achieving the diagnosis. The role of computed tomography (CT) has been defined by Yan et al. who demonstrated that CT characterization and diagnosis of the MPM is possible and further concluded that most patients will have ascites and the predominant areas involved by tumor mass are the vesical or rectal uterine pouch and the greater omentum[7]. Cytologic analysis of ascites has a low diagnostic yield, due to high cytological diversity of tumor cells and small number of malignant cells that are present in the fluid $[2,8]$. Useful tumour markers implicated in the diagnosis and surveillance of peritoneal mesothelioma are carbohydrate antigen (CA) 125, CA 15-3, serum mesothelin-related protein (SMRP) and osteopontin. However, these are nonspecific biomarkers which could be present in other malignant diseases [2,8].

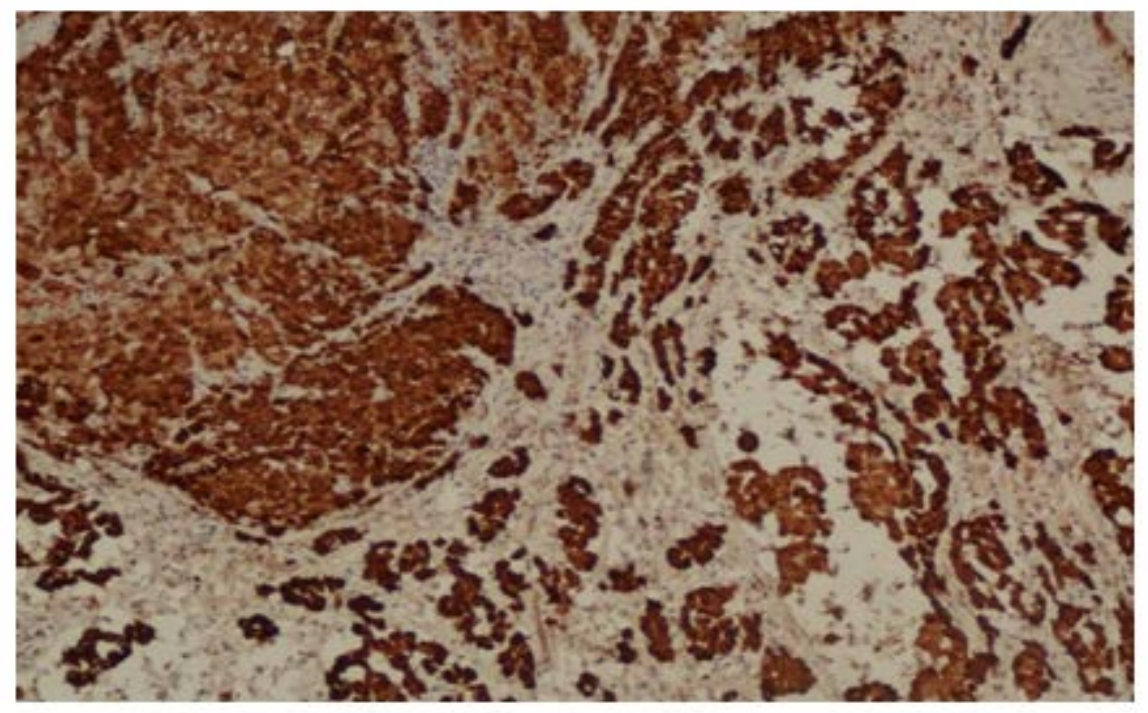

Figure 3. Positive reaction towards mesothelial marker Calretinin

The understanding of its unique tumor biology forms the rationale of treatment strategies designed to control disease progression in the peritoneum. The rarity of the tumor; late presentation of the disease; and the long latency between exposures and diagnosis have made it difficult for clinicians to establish a standard protocol. No standard treatment protocols could be outlined as a variety of modalities attempted either alone or in combination with others. Large studies comparing different therapeutic modalities do not exist in the literature as the disease is rare. Modalities included cytoreductive surgery, hyperthermic intraoperative perfusion of intraperitoneal chemotherapy (HIPEC), early postoperative intraperitoneal chemotherapy (EPIC), whole abdominal radiation and palliative chemotherapy have been reported. However, there is a trend towards aggressive cytoreductive surgery and perioperative intraperitoneal chemotherapy with studies reported a better overall survival (up to 7 years), progression-free survival and near complete palliation of symptomatic ascites in centers performing aggressive surgical debulking followed by hyperthermic intraperitoneal chemotherapy with cisplatin $[9,10,11]$. The rationale behind the hyperthermic intraperitoneal chemotherapy is to deliver high concentrations of cytotoxic agents and hyperthermia directly to the peritoneal tumor, limiting systemic exposure and toxicity [12]. Cisplatin has been chosen as drug of choice because of its minimal regional toxicity, good intraperitoneal pharmacokinetic profile and marked synergy with hyperthermia [10]. Patient selection is important as the prolonged survival must be weighted against the potential morbidity and mortality associated with aggressive cytoreductive surgery [13]. Systemic chemotherapy in recent years using a combination of cisplatin and permetrexed (antimetabolite) have demonstrated an improved median survival of 12-14 month [14,15], compared to the older combination of cisplatin and gemcitabine with a median survival of 6-9 months [16]. However, the median survival of about 1 year is dismal and its role has been limited to palliation and should be considered as the standard of care for patients with unresectable malignant mesothelioma or for patients who are not fit for surgery. Data from Japan looking at the cisplatin based chemotherapy and antimetabolite showed a similar survival benefit [17]. The future of chemotherapy lies with targeted therapies which involve drugs that interfere with specific targeted molecules needed for carcinogenesis and tumor growth. The main advantages are disease-specific treatment with reduced toxicity. However, the results are non-promising with continued research and ongoing trial required [18].

The case illustrates the following points. First, peritoneal mesothelioma is a diagnostic challenge as clinical, radiological and routine laboratory tests are often non-specific. Second, diagnostic laparoscopy and biopsy should be considered as the diagnostic procedure of choice in patients presenting with ascites when laboratory, radiological and endoscopic investigations have proved uninformative.

\section{Conclusions}

Malignant mesothelioma although rare is not obscure in our country. Due to past building practices, many patients have prior asbestos exposure and the possibility of mesothelioma arising in the pleural space or peritoneal cavity is a likely possibility especially due to its long 
latent period. Patients presenting with ascites of unknown origin should undergo diagnostic laparoscopy if all conventional investigations are inconclusive and mesothelioma should be excluded.

\section{Acknowledgement}

We would like to thank the Director General of Health, Malaysia for allowing us to publish this paper.

\section{References}

[1] Papiris SA, Roussos C. Epidemiology and Etiology of Mesothelioma. In: Syrigos KN, Nutting CM, Roussos C, eds. Tumors of the Chest, Berlin Heidelberg: Springer, 2006: 473-480.

[2] Chua TC, Yan TD, Morris DL. Peritoneal mesothelioma: current understanding and management. Can J Surg 2009; 52: 59-64.

[3] Chahinian AP, Pajak TF, Holland JF, Norton L, Ambinder RM, Mandel EM. Diffuse malignant mesothelioma. Prospective evaluation of 69 patients. Ann Intern Med 1982; 96: 746-55.

[4] Weiner SJ, Miandoab. Pathogenesis of malignant pleural mesothelioma and the role of environmental and genetic factors. $\mathrm{J}$ Cancer Res Clin Oncol 2009; 135: 15-27.

[5] Husain AN, Colby TV, Ordonez NG, Krausz T, Borczuk A, Cagle $\mathrm{PT}$, et al. Guidelines for pathologic diagnosis of malignant mesothelioma: a consensus statement from the International Mesothelioma Interest Group. Arch Pathol Lab Med 2009; 133: 1317-1331.

[6] Acherman YTZ, Welch LS, Bromley CM, Sugarbaker PH. Clinical presentation of peritoneal mesothelioma. Tumori 2003; 89: 269-73.

[7] Yan TD, Haveric N, Carmignani CP, Bromley CM, Sugarbaker $\mathrm{PH}$. Computed tomographic characterization of malignant peritoneal mesothelioma. Tumori 2005; 91: 394-400.
[8] Bridda A, Padoan I, Mencarelli R, Frego M. Peritoneal Mesothelioma: A Review. MedGenMed 2007; 9: 32.

[9] Turner KM, Varghese S, Alexander HR. Surgery for peritoneal mesothelioma. Curr Treat Options Oncol 2011; 12: 189-200.

[10] Park BJ, Alexander HR, Libutti SK, Wu P, Royalty D, Kranda KC, et al. Treatment of primary peritoneal mesothelioma by continuous hyperthermic peritoneal perfusion (CHPP). Ann Surg Oncol 1999; 6: $582-590$.

[11] Yan TD, Welch L, Black D, Sugarbaker PH. A systematic review on the efficacy of cytoreductive surgery combined with perioperative intraperitoneal chemotherapy for diffuse malignancy peritoneal mesothelioma. Ann Oncol 2007; 18:827-34.

[12] Alexander HR, Fraker DL. Continuos hyperthermic peritoneal perfusion with cisplatin in the treatment of peritoneal carcinomatosis. Reg Cancer Treat 1995; 8: 2-7.

[13] Yan TD, Edwards G, Alderman R, Marquardt CE, Sugarbaker PH. Morbidity and mortality assessment of cytoreductive surgery and perioperative intraperitoneal chemotherapy for diffuse malignant peritoneal mesothelioma- a prospective study of 70 consecutive cases. Ann Surg Oncol 2007; 14: 515-25.

[14] Castagneto B, Botta M, Aitini E, Spigno F, Degiovanni D, Alabiso $\mathrm{O}$, et al. Phase II study of pemetrexed in combination with carboplatin in patients with malignant pleural mesothelioma (MPM). Ann Oncol 2008; 19: 370-3.

[15] Vogelzang NJ, Rusthoven JJ, Symanowski J, Denham C, Kaukel E, Ruffie P, et al. Phase III study of pemetrexed in combination with cisplatin versus cisplatin alone in patients with malignant pleural mesothelioma. J Clin Oncol 2003; 21: 2636-44.

[16] Fennell DA, Gaudino G, O’Byrne KJ, Mutti L, van Meerbeeck J. Advances in the systemic therapy of malignant pleural mesothelioma. Nat Clin Pract Oncol 2008; 5:136-47.

[17] Kikuchi Y, Watanabe T, Igarashi Y, Sumino Y, Tsuboi K. The chemotherapy of peritoneal malignant mesothelioma. Gan To Kagaku Ryoho 2012; 39: 718-721.

[18] Mott FE. Mesothelioma: A Review. The Ochsner Journal 2012; 12: 70-79. 